\title{
Extraction and diversified application of embroidery insole fabric elements in Hongan, Eastern Hubei
}

\author{
Zhou Yuechao, Li Min, Chen Yu \\ College of industrial design, Hubei University of technology, Wuhan, China
}

\begin{abstract}
By the concept of redesign, the embroidery insole fabric elements of Hongan in Eastern Hubei Province is extracted and applied, and the application research of diversified creative products is carried out. On the premise of inheritance and innovation, the regional characteristics and cultural connotation of Hongan embroidered insole in Eastern Hubei Province are analysed. By understanding the connotation of the elements of Hongan embroidered insole fabric, the composition characteristics of common embroidered insole fabric is grasped, and the traditional fabric themes of Hongan embroidered insole is analysed and arranged; Extract the typical pattern fabric elements that can resonate with modern life, and extract, organize and redesign them; then through integration and reconstruction, carry out diversified application design of creative products. The fabric elements of Hongan embroidered insole are redesigned and applied in fashion, home textile products, clothing, interior decoration, etc. By redesigning, this paper analyses and arranges extracts and redesigns, integrates and reconstructs the elements of embroidered insole fabric in Hongan, Eastern Hubei Province, and studies the application of diversified creative product redesign. This modern process method and diversified application mode is not only the dynamic inheritance of traditional regional culture, but also the innovation and development of folk culture in Eastern Hubei Province. The inheritance and development of art traditional culture has important practical significance.
\end{abstract}

\section{Introduction}

With the development of human civilization, folk cloth art in Eastern Hubei [1], including Hongan embroidery, has undergone changes and is closely related to the quality of life. Hongan embroidery has distinctive artistic characteristics of folk cloth art in Eastern Hubei Province [2]. Hongan embroidery is diverse and rich in content [3]. Hongan embroidered insole is an indispensable daily necessities for people in Hongan. It not only has rich cultural connotation, but also has pleasant texture, excellent production, comfortable and durable [4]. Hongan embroidered insole contains feelings and principles, covering heaven, earth, customs and human feelings; it is widely used in many important moments of life, such as wedding, entrance examination, military service and so on. With the gradual improvement of modern people's living standards, the impact of the progress of contemporary civilization on traditional culture is also gradually obvious. As the production of Hongan embroidered insole is a form of oral transmission, the traditional manual production process cannot be mass produced, facing the situation of the loss of traditional handicraft [5]. Therefore, from the perspective of traditional culture living inheritance [6], this paper, based on the redesign concept [7-8], takes the embroidered insole in
Hongan of Eastern Hubei as an example to study the extraction of fabric art elements and the design and application of diversified creative products.

\section{Extraction of embroidery insole fabric elements from Hongan}

\subsection{Collation and analysis}

Based on the concept of redesign, this paper sorts out and analyzes the embroidery insole fabric elements of Hongan, improves and uses the typical insole fabric elements, inherits and retains the profound connotation implied by the pattern theme of Hongan embroidered insole. The point, line and surface elements of Hongan embroidered insole pattern are embodied by different combinations.

The composition of the embroidered insole in Hongan is usually handed down by the embroidered women, which has a non-strict definition of pattern size and color matching. The composition and color matching of Hongan embroidered shoe mat are determined by the embroidery artists. The technique is at one's heart. It reflects the unique composition characteristics of Hongan embroidery and has typical regional characteristics of folk cloth art culture in Eastern Hubei. The inheritance of cultural connotation behind the pattern is the basis and core of redesign of 
traditional pattern elements of Hongan embroidered insole [9].

There are three types of composition of Hongan embroidered insole, as shown in Figure 1. One is realistic. It is beautified by the natural form of scenery. That is, on the basis of the prototype presented in life, it is processed by refining, strengthening, beautifying and layout. Realistic composition requires both the form and the spirit, and the form similarity reflects the spirit similarity. As shown in Figure 1 (a), "head loving fish" insole has complete fish shaped pattern, distinct gold carp, and both shape and spirit. The other is freehand brushwork. That is, on the basis of understanding the characteristics of natural scenery, embroidery women simplified the composition,

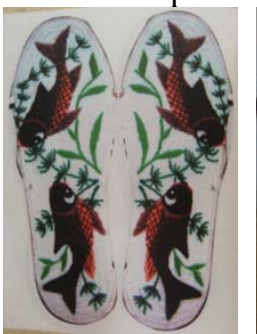

(a)

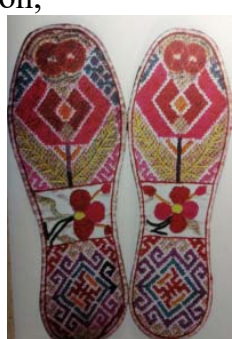

(b) generalized exaggeration, and expressed their ideas. The flower pattern of Hongan embroidered insole is just a kind of moral meaning. Some flower shapes are composed of geometric patterns, such as the insole of "blessing and longevity" in Figure 1 (b), which simplifies the abstract color matching pattern and expresses the meaning of mutual blessing between people. The third is the combination of realism and freehand brushwork. That is, realism and freehand brushwork are used in the composition of a pair of embroidered shoes. As shown in Figure 1 (c), "rich and noble" insole, copper coin realism and peony colorful Phoenix freehand brushwork complement each other [10].

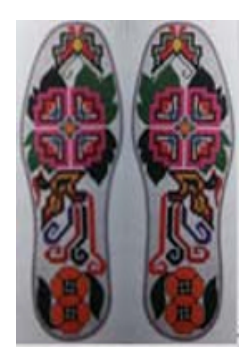

(c)

Figure 1. Embroidered insole pattern of Hongan.

\subsection{Extraction and redesign}

The extraction and redesign of embroidery insole fabric elements in Hongan mainly includes: selection of typical original insole pattern $\rightarrow$ extraction of typical pattern $\rightarrow$ arrangement of pattern $\rightarrow$ redesign of pattern, covering the implication, shape and color of typical insole elements [11]. The pattern features of Hongan embroidered insole are highly geometric and abstract. In the process of pattern extraction, arrangement and redesign of Hongan embroidered insole, the representative patterns and flower shape elements in the insole are described by computer simulation software; the patterns and flower shape elements are highly summarized and sorted; and then the redesign of various variations of patterns and flower shapes is carried out. In this way, the diversified creative product design idea not only retains the meaning of insole pattern and flower shape theme, but also conforms to the original regional style of Hongan embroidery pattern. Figure 2 lists the change graphics in the process of extraction, arrangement and redesign of the animal patterns of some Hongan embroidered insoles; Figure 3 lists the process change graphics of the extraction, arrangement and redesign process of the flower patterns of some Hongan embroidered insoles. 


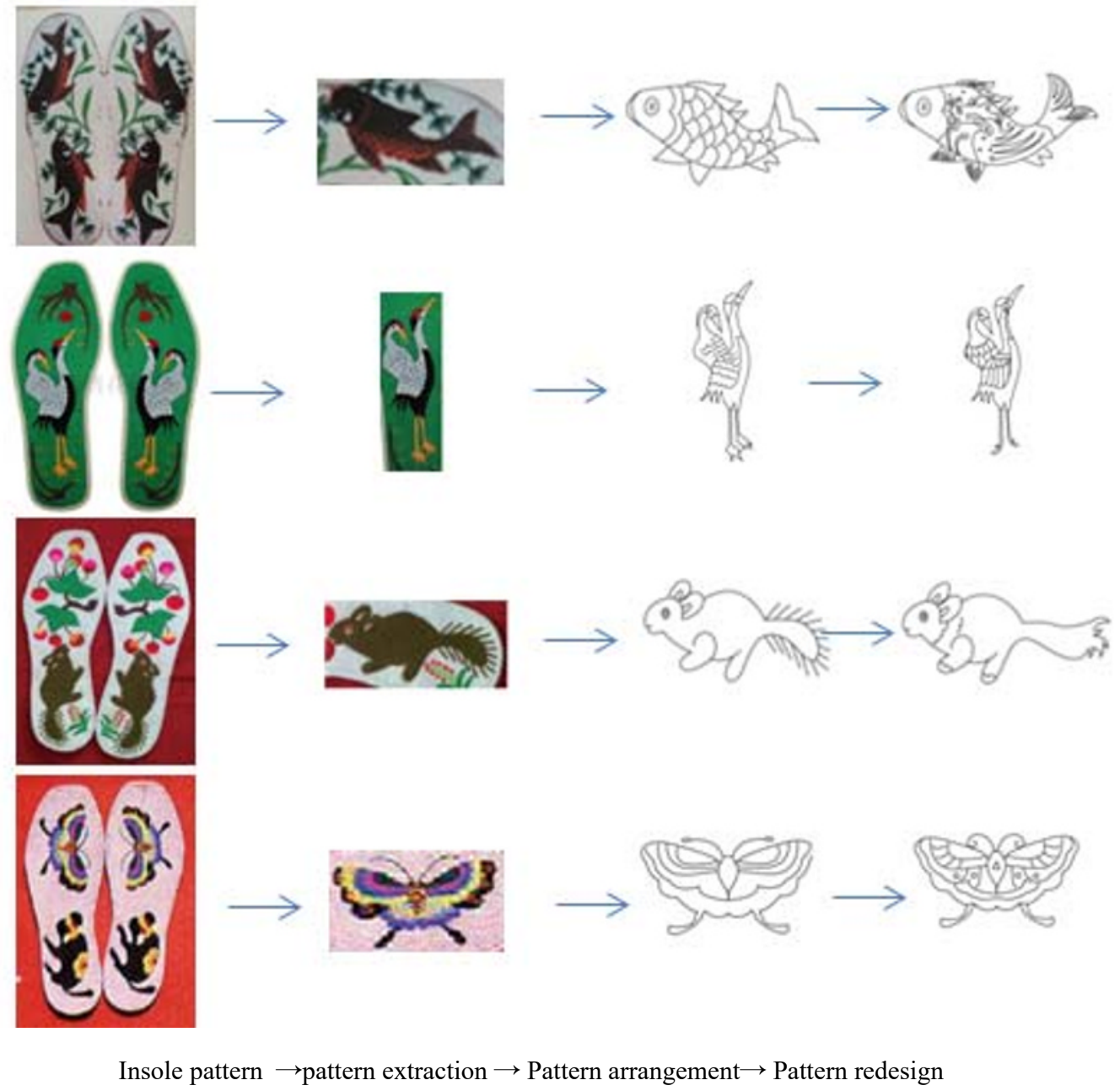

Figure 2. Extraction of animal pattern of Hongan embroidered insole.
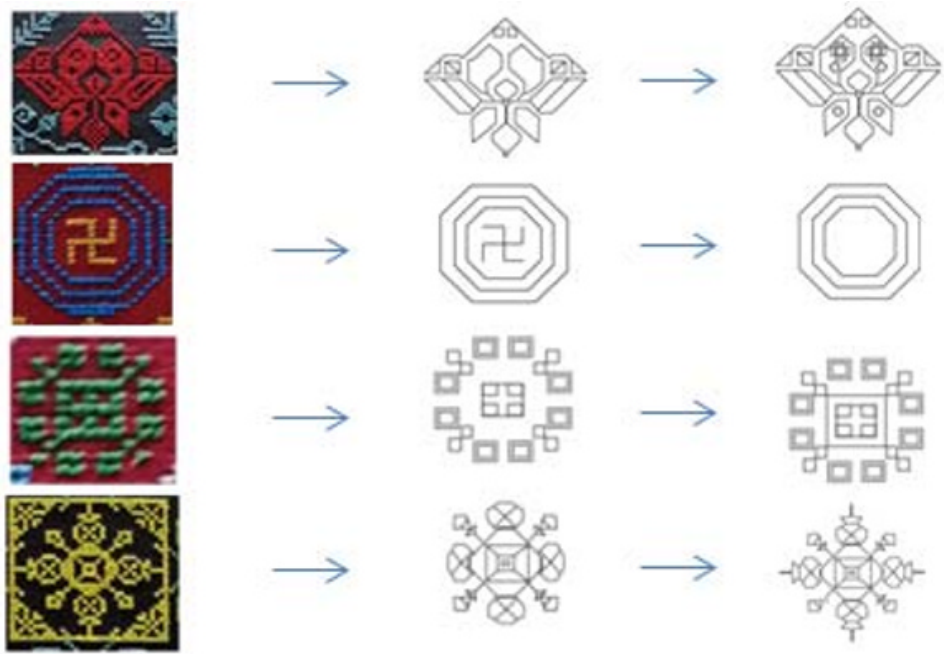

Pattern selection $\rightarrow$ Flower pattern arrangement $\rightarrow$ Redesign of flower pattern

Figure 3. Extraction of flower pattern of Hongan embroidered insole.

\subsection{Integration and reconstruction}

The integration and reconstruction of the typical patterns of Hongan embroidered insole includes the reconstruction of insole pattern and flower shape elements and color reconstruction. In other words, on the basis of retaining the refined and extracted pattern features and flower shape implication, the innovative combination of redesign elements is further carried out to form a transplantable multi creative combination pattern based on Hongan embroidered insole elements. 
The integration and reconstruction of the typical patterns of Hongan embroidered insole includes the reconstruction of insole pattern and flower shape elements and colour reconstruction. In other words, on the basis of retaining the refined and extracted pattern features and flower shape implication, the innovative combination of redesign elements is further carried out to form a transplantable multi creative combination pattern based on Hongan embroidered insole elements.

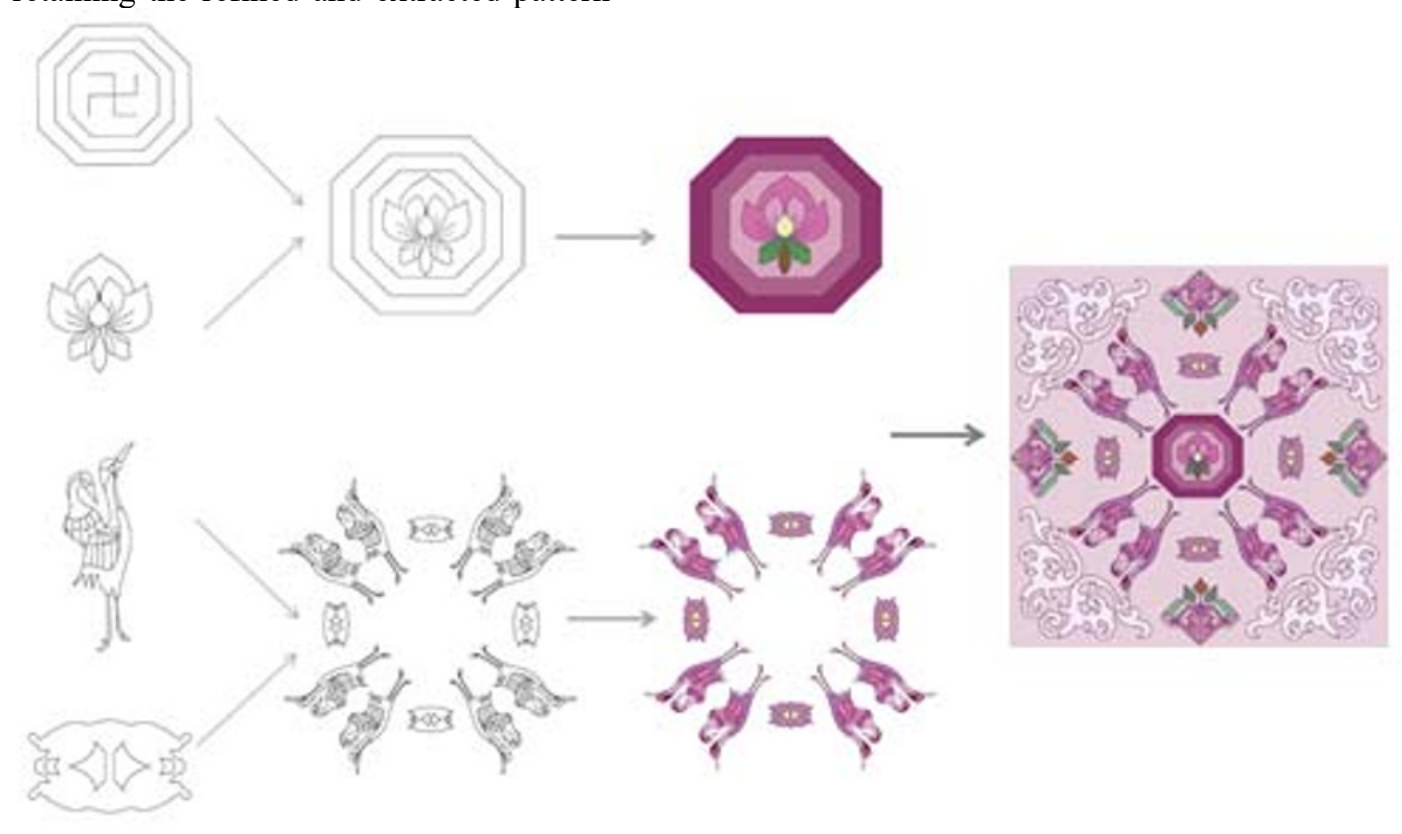

Redesign pattern -- Combinatorial reconfiguration --Color reconstruction--Combination design

Figure 4. Integration and reconstruction of typical pattern elements of "crane stepping on Lotus".

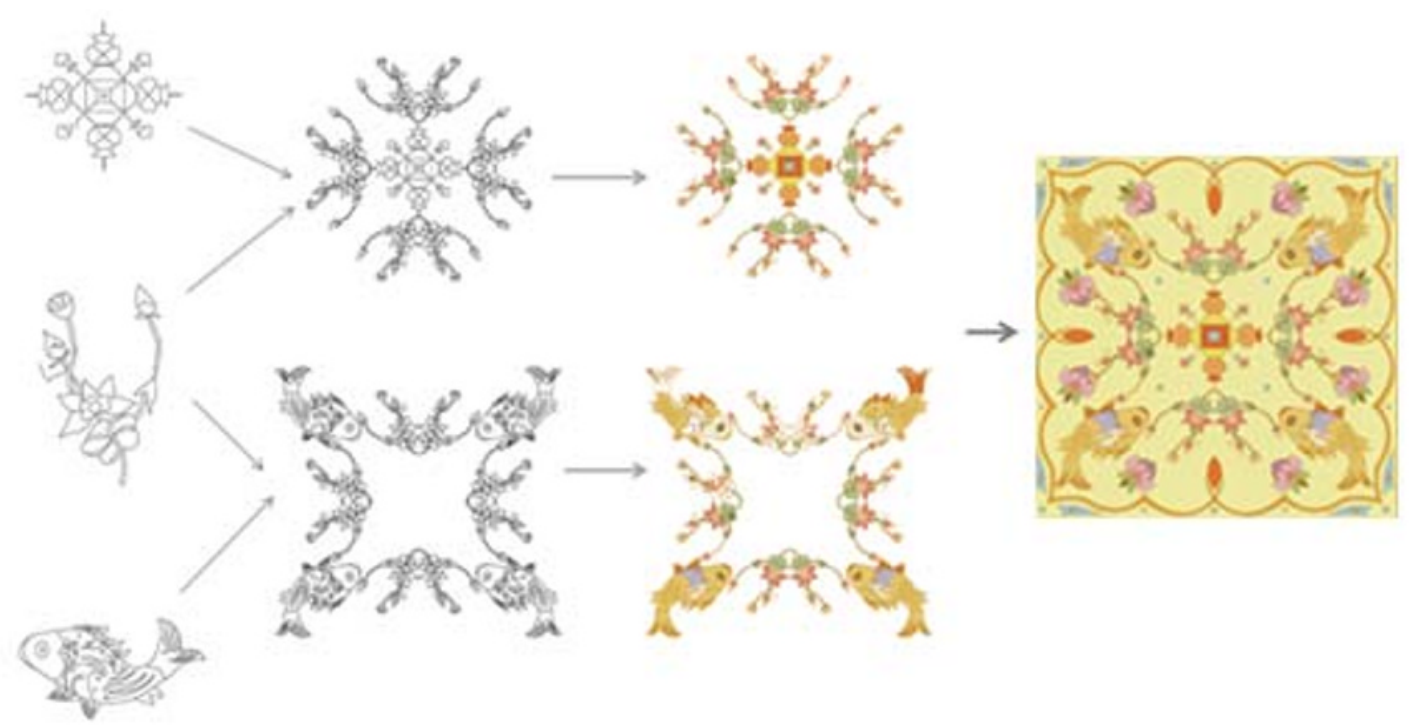

Redesign pattern -- Combinatorial reconfiguration --Color reconstruction--Combination design

Figure 5. Integration and reconstruction of typical pattern elements of "four happy head fish".

\subsubsection{Reconstruction of insole pattern and pattern elements.}

Reconstruction is not the reproduction and copy of embroidery patterns and flower shape elements [12], but based on the concept of redesign, through the use of digital simulation of computer simulation, the pattern and shape of embroidered insole in Hongan are highly abstract and internationalized, which not only retains the original meaning of embroidered insole, but also meets the needs of modern cultural life. The process of combined redesign is to combine the extracted, sorted and redesigned animal and lace patterns into symmetrical patterns or other ways. Figures 4 to Figures 6 show the process of re design combined pattern based on Hongan embroidered insole elements. Figure 4 shows the process of pattern 
reconstruction of embroidered insole of "crane treading Lotus"; Figure 5 shows the process of pattern reconstruction of embroidered insole of "four happy held fish"; and Figure 6 shows the process of pattern reconstruction of embroidered insole of "golden mouse and butterfly playing spring". Reconstruction of insole pattern and pattern elements. Reconstruction is not the reproduction and copy of embroidery patterns and flower shape elements [12], but based on the concept of redesign, through the use of digital simulation of computer simulation, the pattern and shape of embroidered insole in Hongan are highly abstract and internationalized, which not only retains the original meaning of embroidered insole, but also meets the needs of modern cultural life. The process of combined redesign is to combine the extracted, sorted and redesigned animal and lace patterns into symmetrical patterns or other ways. Figures 4 to Figures 6 show the process of re design combined pattern based on Hongan embroidered insole elements. Figure 4 shows the process of pattern reconstruction of embroidered insole of "crane treading Lotus"; Figure 5 shows the process of pattern reconstruction of embroidered insole of "four happy held fish"; and Figure 6 shows the process of pattern reconstruction of embroidered insole of "golden mouse and butterfly playing spring".

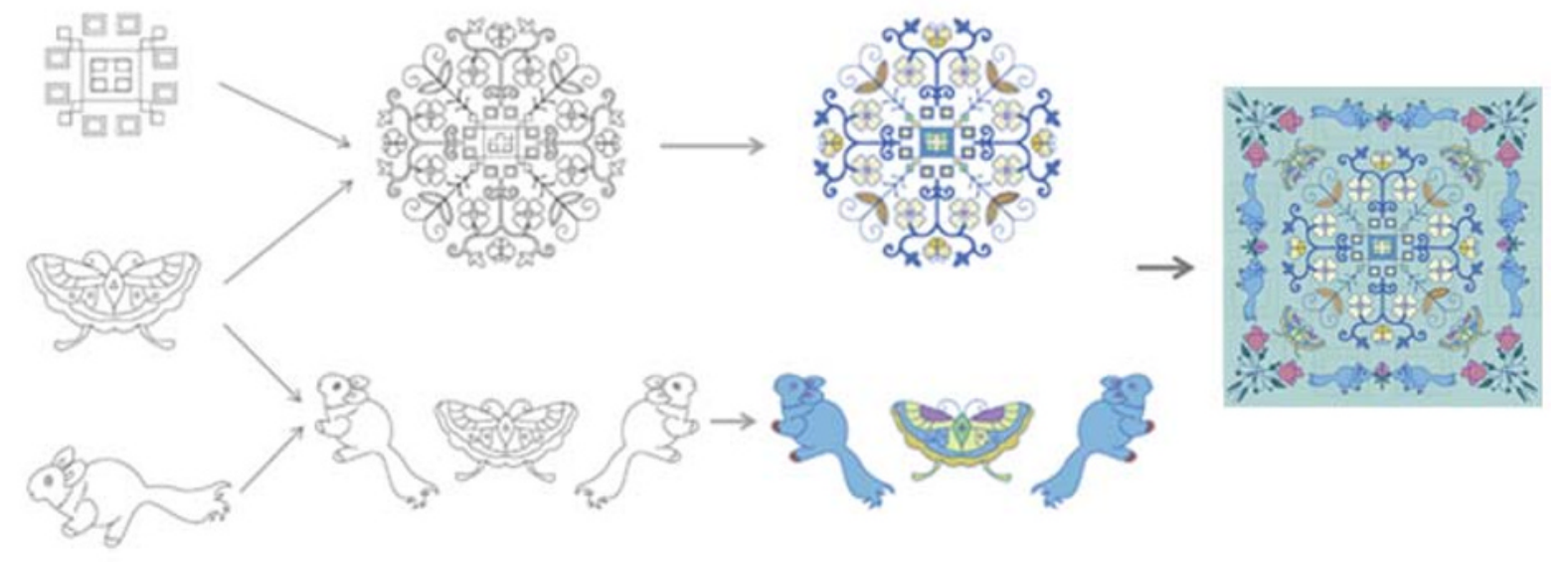

Redesign pattern -- Combinatorial reconfiguration --Colour reconstruction--Combination design

Figure 6. Integration and reconstruction of typical pattern elements of "golden mouse and butterfly make spring".

\subsubsection{The pattern and pattern of insole are reconstructed.}

The colour reconstruction should not only keep the inherent colour characteristics of Hongan embroidered insole pattern and flower shape elements, but also integrate into the popular colours of modern culture. The purpose of colouring is to recreate the diversified creative patterns of insoles on the basis of preserving regional characteristics. In the process of colour matching graphic redesign, the colour matching features of "five elements" of green, white, red, yellow and blue embroidered by Hongan are retained, and the harmony of colours is emphasized. In the process of colour reconstruction of the integrated and reconstructed multi insole elements combination pattern, the colour matching of combination pattern in Figure 6 is gradual harmony colour matching; the colour matching of combination pattern in Figure 4 and Figure 5 is comparative harmonious colour matching.

\section{Hongan embroidered insole fabric elements diversified creative product design and Application}

Hongan embroidered insole in the traditional folk cloth art in Eastern Hubei Province, the fabric design theme involves daily household, animals and plants, Buddhism and Zen, Chu culture, and words and Poems [13]. It seems that there are many types, but the production form is relatively single. Therefore, we can design and develop more fashionable and popular diversified creative products suitable for contemporary people's life by using the extracted insole fabric pattern and flower shape elements from the inheritance and diversity of product types.

\subsection{Applied to fashion products}

The re designed Hongan embroidered insole fabric element combination pattern, because of its portability, can be widely used, so modern fashion products, design and production of more practical value of creative products. Multiple insole fabric elements combination pattern can be applied to handbags, satchels, backpacks, shoulder bags and so on. As shown in Figure 7, it is a combination of multi Hongan embroidered insole fabric elements. The pattern is transplanted to the portable canvas bag to form a convenient and applicable household creative product with a certain cultural background. 


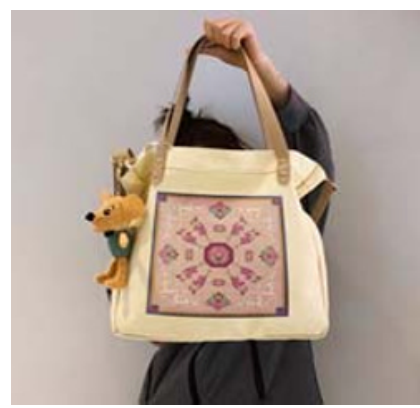

Figure 7. Redesigned handbag.
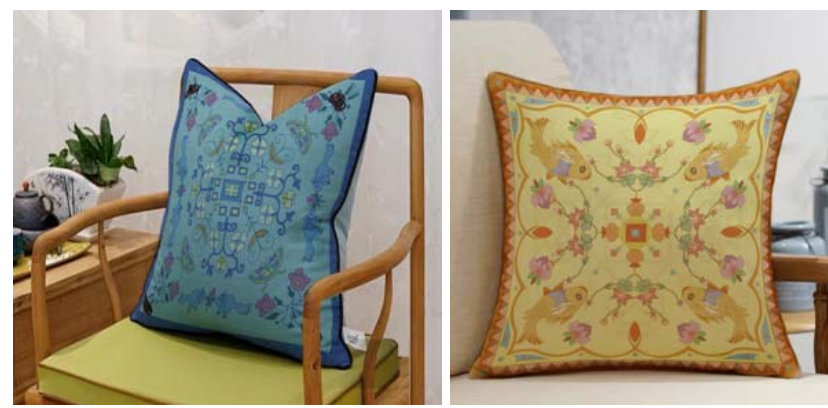

Figure 8. Redesigned pillow.

towel, and tablecloth and so on. As shown in Figure 8, a series of household pillow patterns are redesigned by using the single combination pattern of Hongan embroidered insole fabric elements. As shown in Figure 9, a series of silk scarves are redesigned by using the combination pattern of Hongan embroidered insole fabric elements.
The redesigned Hongan embroidered insole fabric elements can be combined with various home textile products to develop a series of creative home textile products. It can be applied to curtain, bed sheet and quilt cover, pillow and sofa set, etc. Single combination pattern can be applied to pillow, silk
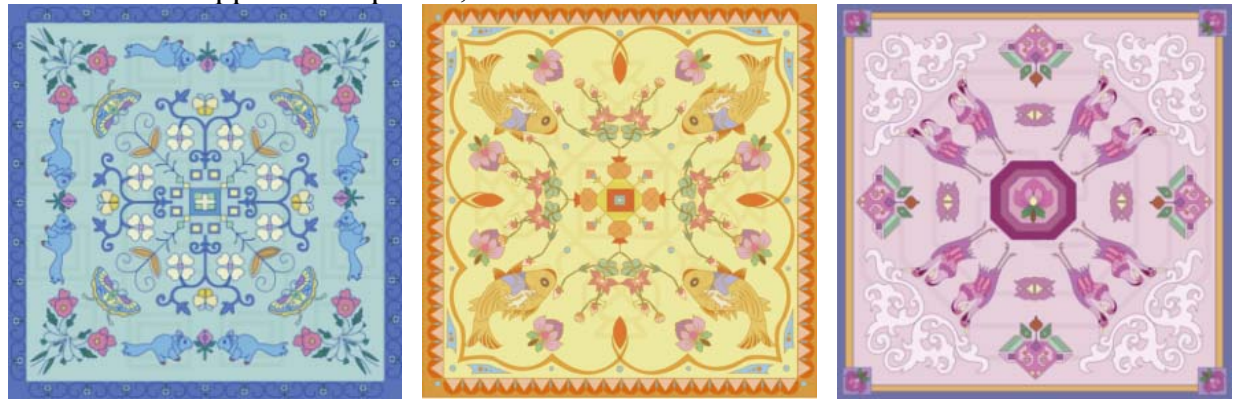

Figure 9. Redesigned silk scarf. subsidies, it can be mixed and matched with modern clothing such as windbreaker, suit, dress, etc. As shown in Figure 10, the effect picture of red an embroidered insole combined with edge flower elements, as well as animal patterns such as golden mouse and crane, is applied to clothing. It is clear, concise and generous.
The combination pattern of Hongan embroidered insole fabric elements can also be used in modern clothing design. Through partial embellishment, animals, plants and flower patterns are partially applied to popular clothing, and can be selectively applied to T-shirts, coats, skirts, shoes and hats; through splicing or
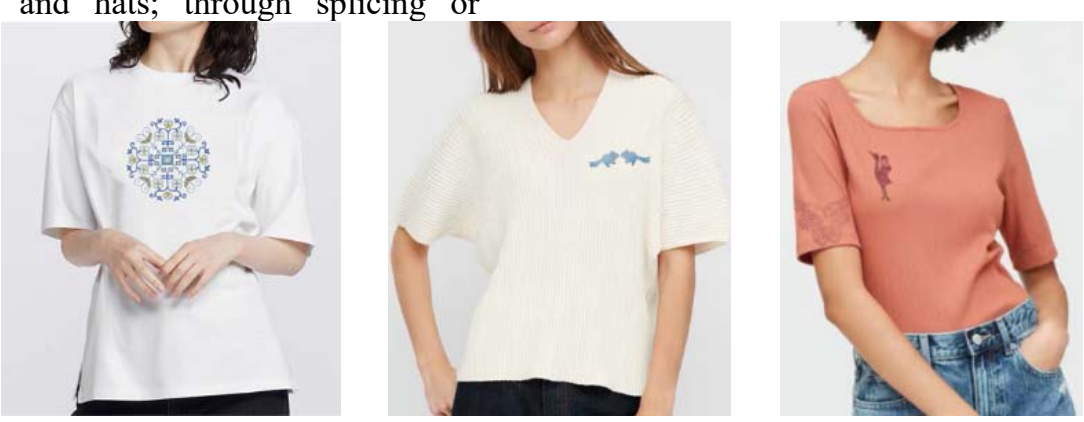

Figure 10. Redesigned clothing patterns.

\subsection{Applied to interior decoration design products}

The re designed Hongan embroidered insole fabric element combination pattern can also be used in interior decoration design [15]. Interior decoration design can directly transplant the combined pattern of redesign to decorative wallpaper, vase, screen and mural. For example, in Figure 11, the creative design of the combination pattern is applied to the interior 
Vase Decoration design. The color is elegant and soft,

elegant and generous.
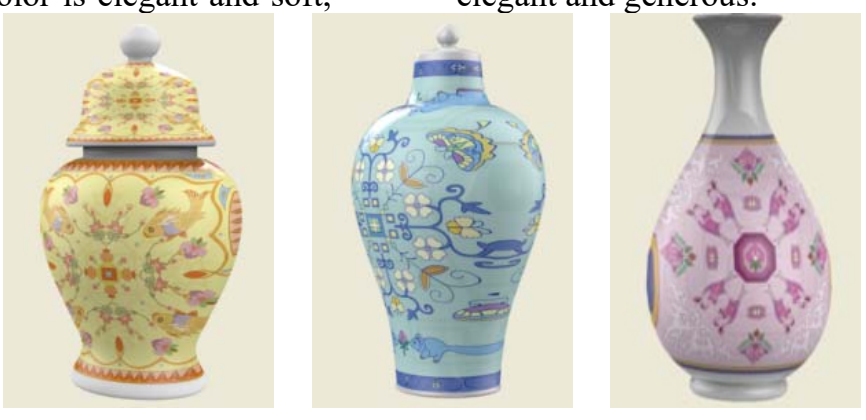

Figure 11. Redesigned decorative vase

\section{Conclusions}

Hongan embroidered insole fabric elements in the folk cloth art of Eastern Hubei Province are rich in patterns and patterns, which carry the emotional sustenance and changes of the times of Hongan people in Eastern Hubei Province. Through the sorting and analysis of the typical patterns and flower shaped cloth art elements of Hongan embroidered insole, extraction, arrangement and redesign, as well as integration and reconstruction, the multiple combination patterns formed from this have strong portability and can be widely used in all aspects of daily life, and then design diversified creative products with regional characteristics. This diversified creative product design idea not only retains the meaning of insole pattern and flower shape theme, but also conforms to the original regional style of Hongan embroidery pattern. Therefore, based on the concept of redesign, taking Hongan embroidered insole as the research object, it has important practical significance to extract the fabric art elements of Hongan embroidered insole and the application of diversified creative products.

\section{Acknowledgments}

This paper is supported by two foundation projects: Research on service design strategy for innovation of folk fabric products in Eastern Hubei Province (20YJA760036), supported by humanities and Social Sciences planning fund of the Ministry of education of China; and Research on collaborative service design based on innovation and development of folk handmade cloth art in Eastern Hubei Province (19D033).

\section{References}

1. Ding bin 2015 Research on the folk cloth art of Eastern (Hubei: Hubei Normal University)

2. Yuan Mengmeng 2019 The artistic characteristics of folk cloth art in Eastern Hubei and the origin of its style Silk vol 56 (04) pp 85-92

3. Huang Lin and Ye Hongguang 2017 Cultural characteristics and design application of Hongan embroidery Tianjin Textile Technology vol 05 pp 4-5

4. Wang Xia 2012 Hongan xiuhuo: a series of intangible cultural heritage of Hubei Province (Hubei: Hubei people's publishing press)

5. Wang Xia 2010 Thinking about the protection and development of "Hongan embroidery activity" A preliminary study on the construction and development of the hometown of folk culture and art in China (Beijing: Beijing shetu Culture Development Co., Ltd) pp 308-310

6. Chen Ruilian and Ye Hongguang 2016 Study on the protection and inheritance of Hongan embroidery Intangible Cultural Heritage Tianjin textile branch vol 4 pp 15-1

7. Jiang min 2013 Iinterpretation and Reflection on product redesign Packaging engineering vol 34 (18) pp 92-95

8. You Wenwen 2020 Study on the secondary design and reuse of garment waste fabric Leather science and engineering vol 30 (3) pp 88-92

9. Zhou Yang 2019 Analysis and design of the pattern of yellow plum in Hubei Province (Hubei: Hubei University of technology)

10. Lu Yanxin 2016 Research on the characteristics of embroidery crafts in Hongan of Hubei Province (Hubei: Wuhan University of technology)

11. Zhang Jingya 2015 Research on Extraction of regional cultural elements and dynamic graphic design based on scene prototype (Hunan: Hunan University)

12. Luoling 2017 Research on the protection of folk embroidery from the perspective of the revitalization of traditional crafts (Hubei: Wuhan Textile University)

13. Ma Jiao 2017 Research on Hongan embroidery in Hubei (Shanghai: Donghua University)

14. Chen Ruilian 2018 Research on the application of folk embroidery in the design of modern women's wear (Hubei: Wuhan Textile University)

15. Longzhou 2017 The application of "craft form qitheism" in the comparative study of Arts and crafts and decorative arts Art observation vol $2 \mathrm{pp}$ $122-123$ 